\title{
Teenage girls with type 1 diabetes have poorer metabolic control than boys and face more complications in early adulthood
}

\author{
Ulf Samuelsson, Johan Anderzen, Soffia Gudbjornsdottir, Isabelle Steineck, Karin Akesson \\ and Lena Hanberger
}

\section{Journal Article}

\section{Tweet}

N.B.: When citing this work, cite the original article.

Original Publication:

Ulf Samuelsson, Johan Anderzen, Soffia Gudbjornsdottir, Isabelle Steineck, Karin Akesson and Lena Hanberger, Teenage girls with type 1 diabetes have poorer metabolic control than boys and face more complications in early adulthood, Journal of diabetes and its complications, 2016. 30(5), pp.917-922.

http://dx.doi.org/10.1016/j.jdiacomp.2016.02.007

Copyright: Elsevier

http://www.elsevier.com/

Postprint available at: Linköping University Electronic Press

http://urn.kb.se/resolve?urn=urn:nbn:se:liu:diva-130403

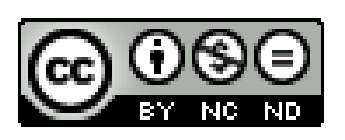





\section{Teenage girls with Type 1 diabetes have poorer metabolic control than boys and face more complications in early adulthood.}

U Samuelsson, J Anderzen, S Gudbjörnsdottir, I Steineck, K Åkesson, L Hanberger

\section{Ulf Samuelsson}

Dept. of Clinical and Experimental Medicine, Division of Paediatrics, Linköping University, Linköping, Sweden

Johan Anderzen

Dept. of Paediatrics, Ryhov County Hospital, Jönköping, Sweden

Soffia Gudbjörnsdottir

Dept. of Medicine, Sahlgrenska Academy, University of Gothenburg, Gothenburg, Sweden

Isabelle Steineck

Dept. of Endocrinology, Copenhagen University Hospital, Hvidovre, Denmark

Karin Åkesson

Dept. of Paediatrics, Ryhov County Hospital, Jönköping, and Futurum - academy for Health and Care, Jönköping County Council, Sweden

Lena Hanberger

Dept. of Medical and Health Sciences, Division of Nursing, Linköping University, Linköping, Sweden

Corresponding author: L Hanberger: Dept. of Medical and Health Sciences, Division of Nursing, Linköping University, Linköping, Sweden. Email: lena.hanberger@liu.se 


\begin{abstract}
Aims: To compare metabolic control between males and females with type 1 diabetes during adolescence and as young adults, and relate it to microvascular complications.

Methods Data concerning 4000 adolescents with type 1 diabetes registered in the Swedish paediatric diabetes quality registry, and above the age of 18 years in the Swedish National Diabetes Registry was used.

Results: When dividing HbA1c values in three groups; < $7.4 \%$ (57 mmol/mol), $7.4-9.3 \%$ (57-78 $\mathrm{mmol} / \mathrm{mol})$ and $>9.3 \%$ (78 $\mathrm{mmol} / \mathrm{mol})$, there was a higher proportion of females in the highest group during adolescence. In the group with the highest HbA1c values during adolescence and as adults, $51.7 \%$ were females, expected value $46.2 \%$; in the group with low HbA1c values in both registries, $34.2 \%$ were females, $\mathrm{p}<0.001$. As adults, more females had retinopathy, $\mathrm{p}<0.05$. Females had higher mean HbA1c values at diagnosis, 11.2 vs $10.9 \%$ (99 vs. $96 \mathrm{mmol} / \mathrm{mol}$ ), $\mathrm{p}<0.03$, during adolescence, 8.5 vs. $8.2 \%$ (69 vs. $66 \mathrm{mmol} / \mathrm{mol}$ ) $\mathrm{p}<0.01$, but not as young adults.

Conclusions: Worse glycaemic control was found in adolescent females, and they had a higher frequency of microvascular complications. Improved paediatric diabetes care is of great importance for increasing the likelihood of lower mortality and morbidity later in life.
\end{abstract}

Keywords HbA1c, type 1 diabetes, gender, microvascular complications 


\section{Introduction}

Type 1 diabetes, TID, is one of the most common chronic diseases of childhood, and the incidence is increasing (1). Previous studies $(2,3)$ have shown that improved glycaemic control, measured as $\mathrm{HbA1c}$, is important in preventing, delaying or slowing the progression of long-term complications. A high proportion of adolescents do not reach treatment targets for glycated haemoglobin, HbA1c (4-6), and there is evidence that metabolic control deteriorates during adolescence. There is a correlation between metabolic control in late childhood and during adolescence (7-9). A gender-dependent difference in metabolic control has also been shown, with females having worse glycaemic control during the clinical course, especially during adolescence (10-13). Girls in the 6-12 year age group presented with higher HbA1c levels than boys and girls of other age groups $(11,13)$. In addition, a higher incidence of diabetic ketoacidosis (DKA), dyslipidemia and growth problems has been reported in female patients (15). In contrast, however, in a review of factors associated with the presence of DKA, no difference due to gender was found (17). A gender difference in residual beta cell function exists when T1D is diagnosed, which has consequences for treatment (18). Pozelli et al. found a more extensive destruction of beta cells in females than in males during puberty (19).

Regarding late complications, there is a higher risk of end-stage renal disease (ESRD) for male than for female patients in Sweden (20). In the EURODIAB study on the association between HbA1c and all-cause mortality, participants who died during follow-up were more often women than men (21). There is also a higher hospitalization rate due to severe diabetic vascular complications among females than among males (22).

Data on children and adolescents with diabetes in Sweden are registered in the Swedish paediatric diabetes quality registry, SWEDIABKIDS. After transition to departments of medicine and primary health care centres, data are continuously followed and registered in a quality registry, NDR, for patients above 18 years of age. In this study, data from both registries were used and provided longitudinal information on glycaemic control, offering possibilities to study gender differences in early glycaemic control and the risk of microvascular complications. 
The aim of this study was to compare metabolic control, measured as HbA1c, between males and females with type 1 diabetes during adolescence and as young adults. How the expected gender differences in metabolic control related to micro- and macroalbuminuria and retinopathy in early adulthood was explored.

\section{Material and Methods}

\subsection{The Swedish paediatric diabetes quality registry, SWEDIABKIDS}

Outpatient attendance data from all Swedish paediatric diabetes centres are registered in SWEDIABKIDS (SWE), established in the year 2000. Since 2007, the registry has included data on almost all (approximately 99\%) children and adolescents with diabetes in Sweden. According to Swedish guidelines, children with diabetes visit the diabetes centre at least four times a year. At these visits, HbA1c and other clinical parameters such as insulin dose, weight, length and blood pressure are measured and reported by trained nurses or physicians online (23).

\subsection{The Swedish National Diabetes Registry, NDR}

The NDR was introduced in 1996 to collect data on clinical characteristics and various risk factors in diabetic patients over 18 years of age at outpatient clinics of departments of medicine and primary health care centres nationwide (24). Two visits a year to a department of internal medicine or a primary health care centre are recommended in the guidelines (25). As with SWE, the completeness of NDR has increased, and in 2013 approximately $90 \%$ of adult patients with type 1 diabetes were included (26).

Both SWE and NDR have the status of a national quality registry (27), and the patients are informed about the registry before they consent to be included. None of the registries collect data on ethnicity, socioeconomic status or educational level, as Swedish legislation does not allow this.

\subsection{Study population}

Data on 4945 patients with T1D, who had been transferred from paediatric to adult care, was collected from SWE. Of these patients, 4239 (85.7\%) had HbA1c values registered both in the years 2011 and 2012 in NDR and were included in the study. Of these, 1960 were females and 2279 were males. 
As the age at the time for diagnosis differed between the patients, the time period for which data were registered varied. Some of the patients were included in SWE for a long period but only for a short period in NDR, and vice versa. The mean age in SWE was $15.0 \pm 1.5$ years (range 13 - 18 years) and the mean age in NDR was $24.8 \pm 3.5$ years (range $21-41$ years); $4.9 \%$ of the patients were above 30 years of age. The mean duration of follow-up was $14.7 \pm$ 5.5 years (range 3 - 38 years). The year of diagnosis of T1D varied from year 1974 to 2009. From 2007, SWE included data on patients from all paediatric clinics in Sweden. During the years 2000 - 2007 patients treated at non-participating clinics were diagnosed with, and treated for diabetes but not included in the registry. As adults they can still have data in NDR. At the time of this study there were about 3520 patients with T1D registered in NDR, who were diagnosed during childhood but not registered in SWE. Furthermore, as more than $90 \%$ of adults with T1D are included in NDR less than $10 \%$ of the patients in SWE are lost to follow-up in NDR.

\subsection{Outcome measures}

All laboratory methods used in Sweden are standardized through EQUALIS (External Quality Assurance in Laboratory Medicine in Sweden). The data on HbA1c obtained from SWE and NDR were derived from capillary blood samples taken and analysed in connection with the visit to the outpatient clinic. All available HbA1c values from the time in SWE were used. In NDR, values from year 2011 and 2012 were used. The mean value was calculated as follows: first the mean HbA1c value for each patient was calculated, and from that the mean HbA1c value for SWE and NDR, respectively, was derived. HbA1c values will be presented as NGSP (\%) and IFCC (mmol/mol) (28). Data on albuminuria, retinopathy, physical activity and smoking habits from NDR was used. Microalbuminuria was defined as urine albumin excretion of 20-200 $\mu \mathrm{g} / \mathrm{min}$, and macroalbuminuria as urine albumin excretion $>200 \mu \mathrm{g} / \mathrm{min}$ in two of three consecutive tests during one year. Retinopathy was assessed locally, i.e. through fundus photography performed by an ophthalmologist, and categorized as yes or no. Physical activity was defined as activity lasting more than 30 minutes and was divided into five levels: never (level 1), less than once/week (level 2), one-two times/week (level 3), three-five times/week (level 4), and daily (level 5).

The national HbA1c target value at the time for the study was $7.4 \%(57 \mathrm{mmol} / \mathrm{mol})$. An HbA1c value above $9.3 \%$ (78 mmol/mol) was considered as a very high value and the patient requires intensified care. Based on this, the HbA1c values were divided into three different 
groups, $<7.4 \%$ (57 mmol/mol) (Low HbA1c), 7.4 - 9.3\% (57 - $78 \mathrm{mmol} / \mathrm{mol}$ ) (Middle HbA1c), and $>9.3 \%$ (78 mmol/mol) (High HbA1c).

\subsection{Statistical analysis}

SPSS $18^{\circledR}$ (SPSS inc., Chicago, IL, USA) was used for the analyses. A student's t-test and one-way analysis of variance (ANOVA) were used. When there were indications of skewed distribution, a Mann-Whitney U-test or Kruskall Wallis test was used. Groups were compared by crosstabs, and chi-square was used for proportions. To test the relationship between HbA1c in SWE and HbA1c during early adulthood in NDR, Spearman's correlation was used. A multivariate logistic regression model adjusted for duration of T1D, age at diagnosis, BMISDS in SWE or NDR, physical activity registered in NDR, and smoking registered in NDR, was used to investigate the risk of high HbA1c levels. A multivariate linear regression model with mean HbA1c in NDR as a dependent variable and mean HbA1c in SWE as independent variables was also used in order to adjust for confounders. A p value $<0.05$, two-sided, was regarded as statistically significant. The results are expressed as mean \pm SD.

\section{Results}

\subsection{Clinical outcomes and treatment}

Girls were younger at diagnosis, had a higher mean HbA1c at diagnosis and during adolescence, and had a higher BMI-SDS during adolescence, 13-18 years of age, than boys (Table 1). In young adults, $>18$ years of age, women had a longer duration of the disease and were more often smokers than men $(\mathrm{p}<0.01)$. In general, there was a positive and significant correlation between mean HbA1c among adolescents 13-18 years of age in SWE and mean HbA1c in NDR in early adulthood $>18$ years of age, both in males (Spearman $r=0.44$, $\mathrm{p}<0.001$ ) and females (Spearman $\mathrm{r}=0.38, \mathrm{p}<0.001$ ). Both unadjusted and even after adjusting for potential confounders, a multivariate linear regression model showed the same pattern, ( $<<0.001)$ (Table 2).

\subsubsection{HbA1c levels and change over time}

As mentioned in the method section the HbA1c values were divided into three groups, Low HbA1c, Middle HbA1c and High HbA1c. It was found that there was a higher proportion of females in the High HbA1c group in adolescents 13-18 years of age $(p<0.001)$ but not as 
young adults $>18$ years of age. The distribution as young adults reflected the gender distribution in the whole study population, in which $46.3 \%$ were females (Table 1). Each patient's HbA1c values during adolescence in SWE were compared by using a paired ttest, with the same patient's values as young adult in NDR. Thirty four percent (139/407) of those in the Low HbA1c group in both registries were females. Of those in the High HbA1c group in both registries a higher proportion were females $(\mathrm{p}<0.001)$ (Figure 1$)$. The difference was due to the gender distribution in the whole study population. Of those in the High HbA1c group as adolescents in SWE but with a lower value as young adults in NDR, 50.9\% (256/503) were females. Of those in the Low HbA1c group in SWE but a higher value in NDR, 45.2\% (245/542) were females. The female patients in the High HbA1c group in both registries had a higher HbA1c at diagnosis than female patients in the Low HbA1c group in both registries, $12.1 \% \pm 2.4$ vs. $10.7 \% \pm 2.4 \%$ (108 $\mathrm{mmol} / \mathrm{m} \pm 27$ vs. $94 \mathrm{mmol} / \mathrm{m} \pm 26$, $\mathrm{p}<0.01)$. This was not found among the male patients.

Furthermore, a logistic regression showed a significantly higher OR of 2.1 for girls to be found in the High HbA1c group in both registries $(\mathrm{p}<0.001)$. When adjusting for age at onset, duration, BMI-SDS, physical activity and smoking, the pattern was the same. This was not found for females in the High HbA1c group in NDR but a lower value in SWE (Table 3). Adjusting for BMI in NDR instead of BMI-SDS in SWE did not change the results.

\subsection{Complications}

More females than males, 57.7\% (909/1576) and 53.4\% (949/1777), respectively, had retinopathy as young adults, $\mathrm{p}<0.05$. There was no obvious difference regarding micro- and macroalbuminuria between the genders; 7.8\% (113/1444) of the females and 6.8\% (115/1679) of the males had microalbuminuria, 2.2\% (31/1397) of the females and 1.9\% (31/1596) of the males had macroalbuminuria.

\subsubsection{HbA1c level in SWEDIABKIDS}

In the Low HbA1c group in SWE, a higher proportion of females than males, although not significant, had retinopathy as young adults. There were no differences related to smoking habits or micro- or macroalbuminuria.

In the High HbA1c group in SWE the proportion of females with micro- and macroalbuminuria as well as retinopathy was higher but not significant. There were significantly more smokers among females, 34.2\%, than among males, $22.6 \%(\mathrm{p}<0.001)$ in this HbA1c group. 


\subsubsection{HbA1c level in NDR}

In the low HbA1c group in NDR, the frequency of retinopathy was higher in females than in males, $46.5 \%$ and $40.4 \%$, respectively, $\mathrm{p}<0.05$. Also in the High HbA1c group in NDR, the frequency of retinopathy was higher in females than in males; $69.9 \%$ and $61.5 \%$, respectively, $\mathrm{p}<0.05$. No significant difference between gender regarding albuminuria was found.

\subsubsection{HbA1c levels in both registries}

Gender differences were seen between the Low HbA1c group and the High HbA1c group, respectively, in both registries. Fewer females than males the Low HbA1c group, although not significant, had albuminuria and retinopathy as young adults. In the High HbA1c group significantly more females $(23.8 \%)$ than males $(11.2 \%)$ had microalbuminuria $(\mathrm{p}<0.04)$ whereas the differences for macroalbuminuria and retinopathy were less obvious. Fewer females than males the Low HbA1c group smoked although the difference was not significant. In the High HbA1c group, $47.4 \%$ of the females were smokers and $29.8 \%$ of the males $(\mathrm{p}<0.01)$.

\section{Discussion}

\subsection{Principal findings}

Diabetes quality registries enable us to study clinical data and care outcomes by providing nationwide population-based data with high ascertainment rates. A quality registry makes it possible to continuously follow quality indicators and results, and to identify areas that need improvement.

This large population-based study using national quality registry data from childhood and adolescence and young adults shows a clear gender difference, with girls presenting poorer metabolic control, measured as HbA1c.

Contrary to Viswanathan et al. (13), but in line with Hochhauser et al. (11) and earlier studies from our group (29, 30), we found higher levels of HbA1c at diagnosis in girls than in boys. The gender difference, with higher mean values of HbA1c in girls, persists during adolescence but not during early adulthood.

\subsection{Comparison with other studies}

Higher HbA1c in girls during clinical follow-up in adolescents is found in many studies with smaller sample sizes $(11,12,16,29,30)$. There can be several reasons for the findings that girls, to a much higher extent than boys, have poor metabolic control during adolescence. For 
example, differences in hormonal factors between genders during puberty could affect metabolic control (31).The reduction of the hormonal difference between genders in young adults could be one reason why the mean HbA1c levels are more equal between the genders in NDR. Healthy females are less insulin-sensitive than healthy males. Such decreased sensitivity is compensated by increased insulin secretion (32). Some studies have also shown that both HbA1c and insulin dose were significantly higher in female patients $(14,16,33)$. In addition to hormonal factors one reason for this could be that girls, more often than boys, suffer from depression and psychological problems. Insulin dose was not included in this study, however, from the annual reports from SWE we know that there is no clear gender difference in insulin dose in the Swedish paediatric population.

It has been suggested that due to a metabolic memory, there is a beneficial effect of good metabolic control to reduce the risk complications later in life (34-36). This indicates the importance of efforts to reduce HbA1c in adolescent girls.

It is notable that the females, to a greater extent than males, in the group with poor metabolic control in both registries also had the highest HbA1c levels at diagnosis. It is unlikely that hormonal factors alone can explain the HbA1c difference at diagnosis as many of the patients are diagnosed before puberty. Moreover, girls seem to have more disease symptoms when they are diagnosed with T1D, including lower base excess, more pronounced weight loss and lower BMI, together with a higher proportion of other autoimmune diseases $(18,29)$. One can also speculate that this may be an effect of attitudes and that we are less likely to observe symptoms in girls than in boys. Unfortunately, the duration of symptoms at diagnosis is not reported in SWE. The pattern is also complicated as girls have higher C-peptide values at diagnosis (18). Different immune regulation in girls than in boys may partly explain this (31).

Our data shows that females more often displayed retinopathy as young adults than males but we found no differences in the frequency of micro- and macroalbuminuria. Several studies have shown that smoking, poor metabolic control and microalbuminuria predict an increased risk of cardiovascular disease (37-39) and it is notable that in this study there was a correlation between impaired glycaemic control and an increased frequency of smoking as well as micro albuminuria, especially in females. A recent study from Sweden (40), as well as others (41), found that mortality due to cardiovascular disease is significantly higher in females than in males with diabetes. Furthermore, female gender per se has been shown to be a risk factor for severe complications of T1D (22). 
It is well-known that good metabolic control reduces the risk of complications. In our study even in the group of patients with good metabolic control, female gender seemed to be a risk factor as females in this group more frequently than males had retinopathy. There are also well-known conflicting results concerning the gender effect, as some studies report a male dominance in diabetic nephropathy and other vascular complications $(20,38,42)$.

\section{3 Strengths and weaknesses of the study}

The strength of the present study is that both SWE and NDR are national quality registries with high coverage ratios. Furthermore, the study is population-based, including a large number of subjects. The national quality registries provided the opportunity to follow all teenagers with T1D into adulthood. A weakness could be that some paediatric centres did not report data to SWE before 2007. Patients who were treated at these centres and transferred before 2007 could not be included in the study. As these centres were randomly distributed it is unlikely that this caused any selection bias.

\subsection{Conclusions}

In conclusion, the focus of the study was to compare glycaemic control between males and females, and relate it to microvascular complications. Female patients have worse glycaemic control during adolescence and are also at a higher risk of late complications as the poor metabolic control seems to persist in adulthood. Females had a higher frequency of retinopathy and micro albuminuria as adults. In addition, females were more often smokers. Thus, more focus on glycaemic control in female patients is necessary, both in clinical practice and in research. There is also a need to improve early identification of patients at risk of poor metabolic control and consequently at risk of complications in early adulthood, as poor metabolic control during adolescence continues into adulthood. For this reason, paediatric diabetes teams have a major mission and responsibility to treat and support patients to achieve and maintain good metabolic control and to stay non-smokers. Treatment and care also have to be individualized. We might then be able to decrease the mortality rate from cardiovascular causes in young T1D patients.

Funding Both SWE and the NDR are financially supported by the Association of Local Authorities and Regions, SALAR. This study was also supported by the Futurum - academy for Health and Care, Jönköping county council, Sweden. 
Duality of interest The authors declare that there are no conflicts of interest associated with this manuscript.

Contribution statement US and LH took the initiative to the study. US designed and conducted the statistical analysis. US, LH and JA wrote the first draft of the manuscript. All authors edited and reviewed the manuscript and approved the final version. The guarantor of this manuscript is US. 
Table 1. Clinical and treatment parameters in relation to gender and the proportion (\%) of males and females in different HbA1c groups. Mean HbA1c (SWE), mean HbA1c in SWEDIABKIDS. Mean HbA1c (NDR), mean HbA1c in NDR.

\begin{tabular}{|c|c|c|c|}
\hline & $\mathrm{n}$ & $\begin{array}{l}\text { Male } \\
n=1960\end{array}$ & $\begin{array}{l}\text { Female } \\
\mathrm{n}=2279\end{array}$ \\
\hline Age at diagnosis, Years, $( \pm S D)$ & 4239 & $10.3^{*}(4.4)$ & $9.7 *(4.2)$ \\
\hline $\mathrm{HbA} 1 \mathrm{c}$ at diagnosis, $\%( \pm \mathrm{SD}), \mathrm{mmol} / \mathrm{mol}( \pm \mathrm{SD})$ & 4239 & $10.9 x(2.3), 96(26)$ & 11.2 x (2.6), 99 (29) \\
\hline MeanHbA1c (SWE), \% ( \pm SD), $\mathrm{mmol} / \mathrm{mol}( \pm \mathrm{SD})$ & 4239 & $8.2 *(1.4), 66(15)$ & $8.5^{*}(1.5), 69(15)$ \\
\hline MeanHbA1c, (NDR), \% ( \pm SD), $\mathrm{mmol} / \mathrm{mol}( \pm \mathrm{SD})$ & 4239 & $8.4(1.4), 68(15)$ & $8.4(1.4), 69(15)$ \\
\hline BMI-SDS (SWE), ( \pm SD) & 4181 & $0.4 *(1.0)$ & $0.7 *(0.9)$ \\
\hline $\mathrm{BMI}(\mathrm{NDR})( \pm \mathrm{SD})$ & 3486 & $25.1(3.9)$ & $25.3(4.3)$ \\
\hline Duration of diabetes, years ( $\pm S D$ ) & 4239 & $14.4^{*}(5.6)$ & $15.1^{*}(5.4)$ \\
\hline Physical activity (NDR), mean level ( \pm SD) & 3455 & $3.4(1.0)$ & $3.5(1.1)$ \\
\hline Proportion of smokers (NDR), \% & 3759 & 13.9\# & 18.1\# \\
\hline \multicolumn{4}{|l|}{ HbA1c groups SWEDIABKIDS } \\
\hline$<7.4 \%(57 \mathrm{mmol} / \mathrm{mol})$ & 975 & $60.6 \%$ & $39.4 \%$ \\
\hline $7.4-9.3 \%(57-78 \mathrm{mmol} / \mathrm{mol})$ & 2375 & $52.8 \%$ & $47.2 \%$ \\
\hline$>9.3 \%,(78 \mathrm{mmol} / \mathrm{mol})$ & 886 & $48.8 \%$ & $51.2 \%$ \\
\hline \multicolumn{4}{|l|}{ HbA1c groups NDR } \\
\hline$<7.4 \%,(57 \mathrm{mmol} / \mathrm{mol})$ & 986 & $56.8 \%$ & $43.2 \%$ \\
\hline $7.4-9.3 \%(57-78 \mathrm{mmol} / \mathrm{mol})$ & 2432 & $53.3 \%$ & $46.7 \%$ \\
\hline > 9.3\%, $(78 \mathrm{mmol} / \mathrm{mol})$ & 922 & $52.1 \%$ & $47.9 \%$ \\
\hline \multicolumn{4}{|l|}{$\mathrm{p}<0.05$} \\
\hline \multicolumn{4}{|l|}{$\# \mathrm{p}<0.01$} \\
\hline$* \mathrm{p}<0.001$ & & & \\
\hline
\end{tabular}


Table 2. Multivariate linear regression with mean-HbA1c in NDR as dependent and mean HbA1c in SWEDIABKIDS as independent for the whole population (total), males and females respectively. The beta-coefficient relates to the mean-HbA1c value in SWEDIABKIDS. Adjusted value includes age at diagnosis, BMI-SDS in SWEDIABKIDS, smoking and physical activity in NDR

\begin{tabular}{lllll}
\hline & R-square & Beta-coefficient $(95 \% \mathrm{Cl})$ & $\mathrm{t}$ & $\mathrm{p}$ \\
\hline Total & & & & \\
$\quad$ Unadjusted & 0.16 & $0.401(0.363-0.417)$ & 28.6 & 0.001 \\
$\quad \begin{array}{l}\text { Adjusted } \\
\text { Males }\end{array}$ & 0.18 & $0.375(0.333-0.400)$ & 21.5 & 0.001 \\
$\quad$ Unadjusted & 0.18 & $0.428(0.392-0.466)$ & & \\
$\quad \begin{array}{l}\text { Adjusted } \\
\text { Females }\end{array}$ & 0.20 & $0.421(0.379-0.471)$ & 22.7 & 0.001 \\
$\quad$ Unadjusted & 0.14 & $0.37(0.312-0.39)$ & 18.0 & 0.001 \\
Adjusted & 0.15 & $0.324(0.26-0.358)$ & & \\
\hline
\end{tabular}


Table 3. Logistic regression model with odds ratio, OR, for the risk of a mean HbA1c $>78$ $\mathrm{mmol} / \mathrm{mol}$. Variables included in the adjusted OR were duration of type 1 diabetes, age at diagnosis, BMI-SDS in SWE, physical activity registered in NDR and smoking registered in the NDR.

\begin{tabular}{lllllll}
\hline & \multicolumn{3}{l}{ Unadjusted OR with 95\% Cl } & \multicolumn{3}{l}{ Adjusted OR with 95\% Cl } \\
\cline { 2 - 7 } HbA1c $>9.3 \%$ & In both & In SWE & In NDR & In both & In SWE & In NDR \\
$(>78 \mathrm{mmol} / \mathrm{mol})$ & & & & & & \\
\hline Males & 1 & 1 & 1 & 1 & 1 & 1 \\
Females, & $2.1^{*}$ & $1.6^{*}$ & $1.2 \#$ & $2.0^{*}$ & $1.5^{*}$ & 1.18 \\
& $(1.5-2.8)$ & $(1.3-1.9)$ & $(1.01-1.5)$ & $(1.3-2.99)$ & $(1.2-1.9)$ & $(0.94-1.48)$ \\
\hline$*<0.001$ & & & & & & \\
$\#<0.05$ & & & & & &
\end{tabular}




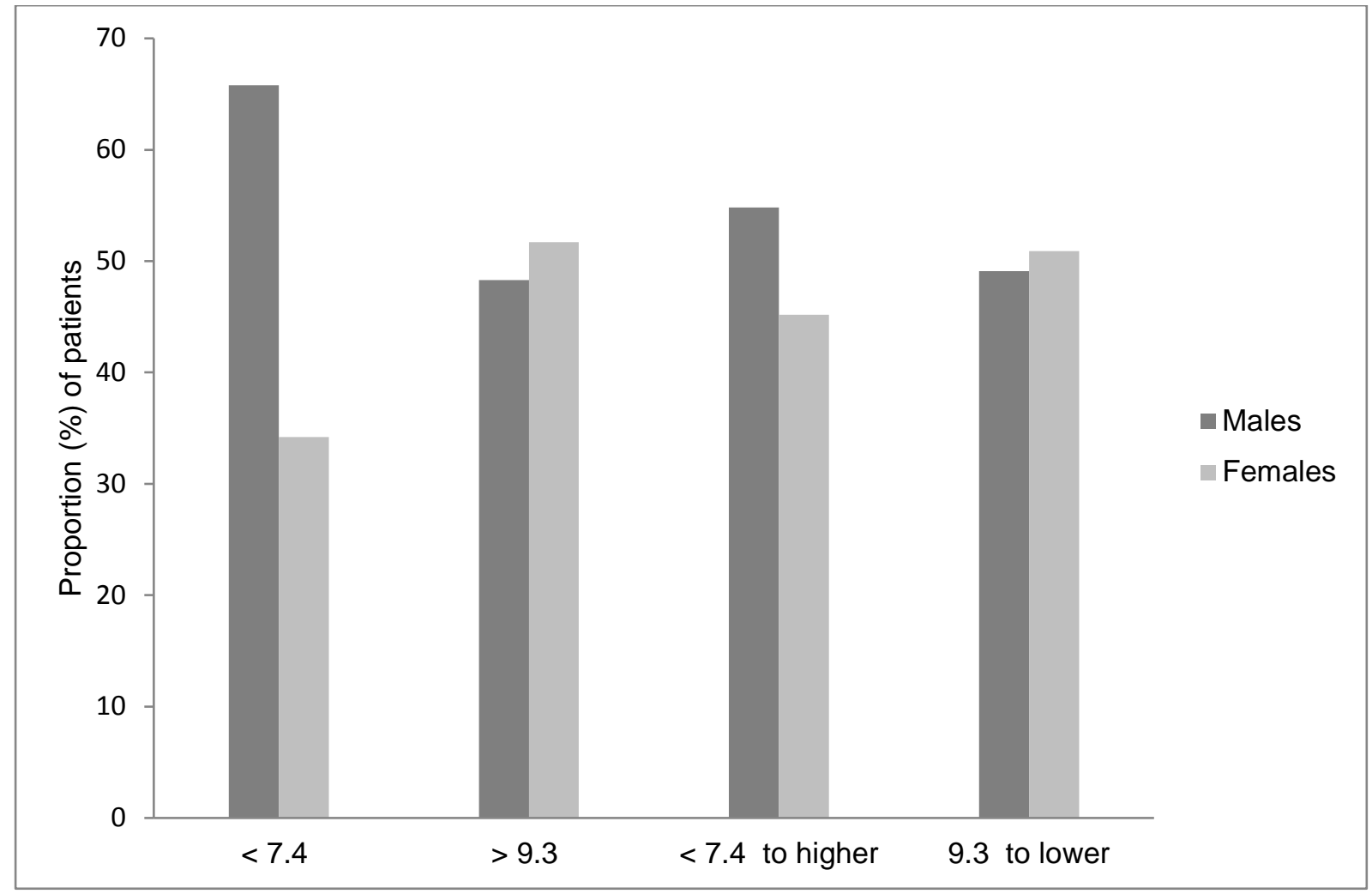

Figure 1. 


\section{Figure legends}

Figure 1. The proportion of males and females in different mean HbA1c groups. <7.4:

patients with HbA1c value < 7.4\% in both registries, > 9.3: patients with HbA1c value > 9.3\% in both registries, 7.4 to higher: patients with $\mathrm{HbA1c}$ value $<7.4 \% \mathrm{mmol} / \mathrm{mol}$ in SWEDIABKIDS but higher HbA1c value in NDR, 9.3 to lower: patients with HbA1c value $>9.3 \% \mathrm{mmol} / \mathrm{mol}$ in SWEDIBAKIDS but lower HbA1c value in NDR. 


\section{References}

1. Diamond Project Group. Incidence and trends of childhood Type 1 diabetes worldwide 1990-1999. Diabet Med 2006; 23 :857-866

2. Diabetes Control and Complications Trial Research Group. The effect of intensive treatment of diabetes on the development and progression of long-term complications in insulin-dependent diabetes mellitus. N Engl J Med 1993; 329: 977-986

3. Nordwall M, Arnqvist HJ, Bojestig M, Ludvigsson J. Good glycaemic control remains crucial in prevention of late diabetic complications - the Linkoping Diabetes Complications Study. Pediatr Diabetes 2009; 10: 168-176

4. Hanberger L, Samuelsson U, Lindblad B, Ludvigsson J. A1C in children and adolescents with diabetes in relation to certain clinical parameters: the Swedish Childhood Diabetes Registry. SWEDIABKIDS. Diabetes Care 2008; 31: 927-929

5. Holl RW, Swift PG, Mortensen HB et al. Insulin injection and metabolic control in an international survey of adolescents with type 1 diabetes over 3 years: results from the Hvidore study group. Eur J Pediatr 2003; 162: 22-29

6. Levine BS, Anderson BJ, Butler DA, Antisdel JE, Brackett J, Laffel LM. Predictors of glycaemic control and short-term adverse outcomes in youth with type 1 diabetes. $\mathrm{J}$ Pediatr 2001; 139: 197-203

7. Cardwell CR, Patterson CC, Allen M, Carson DJ. Northern Ireland Paediatric Diabetes Study Group. Diabetes care provision and glycaemia control in Northern Ireland: a UK regional audit. Arch Dis Child 2005; 90: 468-473

8. Dabadghao P, Cameron FJ. Deteriorating diabetic control during adolescence - do the origins lie in childhood? Diabet Med 2001; 18: 889 - 894

9. Mortensen HB, Hougaard P. Comparison of metabolic control in a cross-sectional study of 2,873 children and adolescents with IDDM from 18 countries. The Hvidore Study Group on Childhood Diabetes. Diabetes Care 1997; 20:714-20

10. Schwab KO, Doerfer J, Marg W, Schober E, Holl RW, DPV Science Initiative and the Competence Network Diabetes mellitus. Characterization of 33488 children and adolescents with type 1 diabetes based on the gender-specific increase of cardiovascular risk factors. Pediatr Diabetes 2010; 11: 357-63

11. Hochhauser CJ, Rapaport R, Shemesh E, Schmeidler J, Chemtob CM. Age at diagnosis, gender and metabolic control in children with type 1 diabetes. Pediatr Diabetes 2008; 9: 303-307. 
12. Gerstl EM, Rabl W, Rosenbauer J et al. Metabolic control as reflected by HbA1c in children, adolescents and young adults with type 1 diabetes mellitus: combined longitudinal analysis including 27,305 patients from 207 centres in Germany and Austria during the last decade. Eur J Pediatr 2008; 167: 447- 453

13. Viswanathan V, Sneeringer MR, Miller A, Eugster EA, DiMeglio LA. The utility of haemoglobin A1c at diagnosis for prediction of future glycaemic control in children with type 1 diabetes. Diabetes Res Clin Pract 2011; 92:65-68

14. Setoodeh A, Mostafavi F, Hedayat T. Glycaemic control in Iranian Children with Type 1 diabetes Mellitus: Effect of gender. Indian J Pediatr 2012; 79: 896-900

15. Samuelsson U, Steineck I, Gubbjornsdottir S. A high mean-HbA1c value 3-15 months after diagnosis of type 1 diabetes in childhood is related to metabolic control, macro albuminuria, and retinopathy in early adulthood - a pilot study using two nation-wide population based quality registries. Pediatr Diabetes 2014; 15: 229-235

16. Setoodeh A, Fereydoun M, Rabbani A, Hedayat T. Female sex as a risk factor for glycaemic control and complications in Iranian patients with type one diabetes mellitus. Iranian J Pediatr 2011; 21: 373-378

17. Usher-Smith JA, Thompson MJ, Sharp SJ, Walter FM. Factors associated with the presence of diabetic ketoacidosis at diagnosis of diabetes in children and young adults: a systematic review. BMJ 2011; 343:d4092

18. Samuelsson U, Lindblad B, Carlsson A et al. Better Diabetes Diagnosis study group. Residual beta cell function at diagnosis of type 1 diabetes in children and adolescents varies with gender and season. Diabetes Metab Res Rev 2013; 29: 85-9

19. Pozzilli P, Mesturino CA, Crino A, Gross TM, Jeng LM, Visalli N, IMDIAB Group. Is the process of beta-cell destruction in type 1 diabetes at time of diagnosis more extensive in females than in males? Eur J Endocrinol 2001; 145 :757-61

20. Möllsten A, Svensson M, Waernbaum I et al. Diabetes Incidence Study in Sweden; Swedish Renal Registry. Cumulative risk, age at onset, and sex-specific differences for developing end-stage renal disease in young patients with type 1 diabetes: a nationwide population-based cohort study. Diabetes 2010; 59 :1803-1808

21. Schoenaker DA, Simon D, Chaturvedi N, Fuller JH, Soedamah-Muthu SS EURODIAB Prospective Complications Study Group. Glycaemic control and all-cause mortality risk in type 1 diabetes patients: the EURODIAB Prospective Complications Study. J Clin Endocrinol Metab 2014; 99: 800-807 
22. Dahlquist G, Möllsten A, Källén B, Swedish Childhood Diabetes Study Group. Hospitalization for vascular complications in childhood onset type 1 diabetes-effects of gender and age at onset. Acta Paediatr 2008; 97: 483-488

23. The Swedish paediatric diabetes quality registry, Swediabkids. Available at https://swediabkids.ndr.nu/ . Accessed 27-11-2015.

24. The National Diabetes Registry, NDR. Available at http://www.ndr.nu Accessed 2711-2015.

25. National guidelines for diabetes care. Available at http://www.socialstyrelsen.se/publikationer2014/2014-619/Sidor/default.aspx Accessed 27-11-2015.

26. National Diabetes Registry, Annual Report 2013. Available at https://www.ndr.nu/pdf/Annual_Report_NDR_2013.pdf Accessed 26-06-2015.

27. Swedish Association of Local Authorities and Regions. Available at http://skl.se/tjanster/englishpages.411.html_Accessed 27-11-2015

28. Hanas R, John G. 2010 consensus statement on the worldwide standardization of the haemoglobin A1c measurement. Diabetes Care 2010; 33: 1903-1904

29. Hanberger L, Akesson K, Samuelsson U. Glycated haemoglobin variations in paediatric type 1 diabetes: the impact of season, gender and age. Acta Paediatrica 2013; 103: 398-403

30. Akesson K, Hanberger L, Samuelsson U. The influence of age, gender, insulin dose, BMI, and blood pressure on metabolic control in young patients with type 1 diabetes. Paediatric diabetes 2014 Oct 1. doi: 10.1111/pedi.12219. [Epub ahead of print]

31. Hoffman RP, Vicini P, Sivitz WI, Cobelli C. Pubertal adolescent male-female differences in insulin sensitivity and glucose effectiveness determined by the one compartment minimal model. Pediatr Res 2000; 48: 284-388

32. Ahmed ML, Conners MH, Drayer NM, Jones JS, Dunger DB. Pubertal growth in IDDM is determined by HbA1c levels, sex, and bone age. Diabetes Care 1998; 21: 831835

33. Knerr I, Hofer SE, Holterhus PM et al. Prevailing therapeutic regimes and predictive factors for prandial insulin substitution in 26687 children and adolescents with Type 1 diabetes in Germany and Austria. Diab Med 2007; 24: 1478-1481

34. Diabetes Control and Complications Trial (DCCT)/Epidemiology of Diabetes Interventions and Complications (EDIC) Research Group. Effect of Intensive Diabetes 
Therapy on the Progression of Diabetic Retinopathy in Patients with Type 1 Diabetes: 18 Years of Follow-up in the DCCT/EDIC. Diabetes. 2015; 64(2):631-42. doi: 10.2337/db14-0930. Epub 2014 Sep 9.

35. White NH, Sun W, Cleary PA; Diabetes Control and Complications Trial (DCCT)/Epidemiology of Diabetes Interventions and Complications (EDIC) Research Group. Effect of prior intensive therapy in type 1 diabetes on 10-year progression of retinopathy in the DCCT/EDIC: comparison of adults and adolescents. Diabetes 2010: 59:1244-1253

36. White NH, Cleary PA, Dahms W, Goldstein D, Malone J, Tamborlane WV; Diabetes Control and Complications Trial (DCCT)/Epidemiology of Diabetes Interventions and Complications (EDIC) Research Group. Beneficial effects of intensive therapy of diabetes during adolescence: outcomes after the conclusion of the Diabetes Control and Complications Trial (DCCT). J Pediatr 2001;139:804-81

37. Mulhauser I, Overmann H, Bender R, Jorgens V, Berger M. Predictors of mortality and end-stage diabetic complications in patients with type 1 diabetes on intensified therapy. Diab Med 2000; 17: 727-734

38. Rossing P, Hougaard P, Borch-Johnsen K, Parving HH. Predictors of mortality in insulin-dependent diabetes: 10-year observational follow-up study. BMJ 1996; 313 : 779-784

39. Lind M, Bounias J, Olsson M, Gudbjornsdottir S, Svensson A-M, Rosengren A. Glycaemic control and incidence of heart failure in 20985 patients with type 1 diabetes: an observational study. Lancet 2011; 378: 140-146

40. Lind M, Svensson A-M, Kosiborod M, et al. Glycaemic control and excess mortality in type 1 diabetes. N Engl J Med 2014; 371: 1972-1982

41. Laing SP, Swerdlow AJ, Slater SD et al. Mortality from heart disease in a cohort of 23,000 patients with insulin-treated diabetes. Diabetologia 2003; 46: 760-765

42. Raile K, Galler A, Hofer S et al. Diabetic nephropathy in 27,805 schoolchildren, adolescents and adults with type 1 diabetes: effect of diabetes duration, A1c, hypertension, dyslipidemia, diabetes onset, and sex. Diabetes Care 2007; 30: 25232528 